\title{
DETERMINAÇÃO DO COEFICIENTE DE DISTRIBUIÇÃO (Kd) DE BENZO(A)PIRENO EM SOLO POR ISOTERMAS DE SORÇÃO
}

\author{
Adriana D'Agostinho e Marlene Flues* \\ Centro de Química e Meio Ambiente, Instituto de Pesquisas Energéticas e Nucleares, Cidade Universitária, \\ CP 11049, 05422-970 São Paulo - SP, Brasil
}

Recebido em 4/3/05; aceito em 24/10/05; publicado na web em 31/3/06

\begin{abstract}
DETERMINATION OF THE DISTRIBUTION COEFFICIENT (Kd) OF BENZO(A)PYRENE IN SOIL USING SORPTION ISOTHERMS. Leaking of diesel oil from gas stations is frequent in Brazil. The presence of polycyclic aromatic hydrocarbons (PAHs), which are highly toxic is an indication of contamination by heavy hydrocarbons from diesel oil. Here were present the determination of the distribution coefficient (Kd) of benzo(a)pyrene (the most carcinogenic of the PAHs) in tropical soils using the sorption isotherm model. The sorption curves acquired for benzo(a)pyrene were of the S-type, probably due to the water/methanol experimental conditions. The sorption curves allowed calculation of the distribution coefficient $(\mathrm{Kd})$. The experimental $\mathrm{Kd}$ values were lower than those calculated from literature Koc values (partition coefficient normalized by organic carbon), due mainly to the cosolvency effect and the percentage of organic matter and clay in soil.
\end{abstract}

Keywords: benzo(a)pyrene; sorption isotherm; soil.

\section{INTRODUÇÃO}

São freqüentes os problemas das indústrias de petróleo decorrentes de vazamentos, derrames e acidentes ambientais envolvendo combustíveis fósseis e seus derivados.

Atualmente, com o grande crescimento do número de postos de combustíveis no Brasil a partir da década de 70, verificou-se também uma elevada preocupação com essa problemática ambiental e, em especial, com o grande potencial de risco de contaminação das águas subterrâneas decorrentes de derramamentos de combustíveis.

Os HPAs têm recebido mais atenção desde que foram encontrados no solo pela primeira vez por Blümer, em $1961^{1}$, devido ao elevado impacto no meio ambiente e do seu potencial carcinogênico. A concentração de benzo(a)pireno (BAP) no solo tem aumentado consideravelmente devido à ampliação de seu uso industrial no último século, como o aumento do trânsito nos grandes centros urbanos e aos freqüentes vazamentos de diesel provenientes de postos de combustíveis.

A importância do estudo do comportamento de BAP em solo se deve à ocorrência de contaminações de aqüíferos e, conseqüentemente, à contaminação de água utilizada para consumo humano. Para tanto, pesquisas das propriedades físicas e químicas desse composto, das características do solo e do coeficiente de distribuição (Kd) são necessários.

Os objetivos deste trabalho foram: determinação do coeficiente de distribuição $(\mathrm{Kd})$ através de isotermas de sorção; comparação dos dados experimentais de $\mathrm{Kd}$ com dados da literatura e discussão dos principais motivos da variação dos valores de Kd experimentais quando comparados com a literatura.

Uma forma de avaliar-se a sorção e a mobilidade do BAP no solo é através dos valores de Kd (coeficiente de distribuição - Equação 1) , que também pode ser denominado de coeficiente de partição; Koc (coeficiente de partição correlacionado com substâncias orgânicas - Equação 2); e Kow (fator de hidrofobicidade - Equação 3).

*e-mail: mflues@ipen.br
$\mathrm{Kd}=\frac{\mathrm{S}}{\mathrm{C}}\left(\mathrm{L} \mathrm{kg}^{-1}\right)$

onde: $\mathrm{S}=$ massa do contaminante na fase sólida do solo $\left(\mathrm{mg} \mathrm{kg}^{-1}\right)$; $\mathrm{C}=$ massa do contaminante na fase líquida $\left(\mathrm{mg} \mathrm{L}^{-1}\right)$.

O parâmetro $\mathrm{Kd}\left(\mathrm{L} \mathrm{kg}^{-1}\right)$ é uma importante ferramenta na estimativa do potencial de sorção do contaminante dissolvido em contato com o solo. Quanto maior o Kd, maior a tendência do contaminante ficar adsorvido ao solo ou sedimento ${ }^{2}$.

Devido à importância do carbono orgânico presente no solo no processo de sorção e distribuição de compostos orgânicos, o coeficiente de distribuição (Kd) é geralmente expresso por Koc; onde Koc é o coeficiente de partição do contaminante na fração orgânica do solo.

$\mathrm{Koc}=\frac{\mathrm{Kd}}{\mathrm{foc}}$

onde: Koc = coeficiente de partição normalizado pelo carbono orgânico ( $\mathrm{L} \mathrm{kg}^{-1}$ substância orgânica); foc = fração de carbono orgânico (kg substância orgânica/kg solo seco).

Koc é, portanto, o coeficiente de partição do contaminante entre solo-água corrigido pela matéria orgânica do solo. A força de sorção entre BAP e o solo é medida pelo coeficiente de partição Koc, que depende das propriedades físico-químicas deste contaminante e da porcentagem de carbono orgânico do solo.

Os valores de Koc são normalmente determinados com base nos valores de Kd e corrigidos pela fração orgânica do solo. No manual de valores de Koc para solo ("Soil Screening Guindance”, EPA) ${ }^{3}$ são apresentadas amplas faixas de valores de Koc para um grande número de compostos orgânicos, que foram obtidas, em sua maioria, por isotermas de sorção. Já em um relatório holandês ${ }^{4}$, a relação entre Kow e Koc é freqüentemente utilizada para estabelecerem-se os valores de Koc. Esta forma simplificada aparentemente representa bem a sorção de contaminantes orgânicos ao solo.

O coeficiente de partição octanol-água, Kow, é outro importante coeficiente para a mobilidade do BAP. Kow é definido como a relação da concentração em equilíbrio de um contaminante orgânico na fase octanol em relação à concentração do contaminante na fase aquosa. 
O valor de Kow pode ser usado para estimar o comportamento de compostos orgânicos hidrofóbicos que não interagem eletricamente com a superfície do solo. O Kow é um coeficiente muito útil para previsão de outros parâmetros como Koc, quando este não se encontra disponível na literatura ${ }^{5}$.

Kow $=\underline{\text { Concentração em equilíbrio do composto químico em octanol }}$

Concentração do composto químico em água

Kow é uma medida que define a hidrofobicidade de um composto orgânico e é um parâmetro comumente utilizado na área ambiental. Koc está fortemente correlacionado com Kow, em uma relação linear expressa pela Equação 4:

$\log \mathrm{Koc}=\mathrm{a} \log \mathrm{Kow}+\mathrm{b}$

onde a e b são coeficientes relacionados com os compostos e tipo de matéria orgânica sobre o solo.

\section{Importância da determinação do coeficiente de distribuição (Kd) para os estudos de análises de risco}

O processo que consiste de tomada de decisões para avaliação e resposta a um vazamento de petróleo (ou produtos químicos em geral), baseado na proteção da saúde humana e do meio ambiente é denominado Ações Corretivas Baseadas em Risco (ACBR) ${ }^{6}$.

Esse processo é, na verdade, um procedimento que integra métodos de avaliação de exposição e de risco, e modelos matemáticos de transporte de contaminantes, fornecendo subsídios ao processo de tomada de decisão relacionada à alocação de recursos, à urgência de ações corretivas, à necessidade de remediação, aos níveis de remediação aceitáveis e às alternativas tecnológicas aplicáveis.

Nos Estados Unidos esta metodologia é denominada "RBCA Risk-Based Corrective Action" (ASTM-PS-104)7, onde muitos estados modificaram sua estrutura adequando os parâmetros a suas necessidades. Em outros países, são utilizadas metodologias semelhantes à da ASTM-PS-104, baseando-se no mesmo conceito, porém com equações diferentes (ex.: C-Soil: metodologia holandesa). No Brasil, pela falta de consenso na utilização de um único método, a Câmara Ambiental do Comércio de Derivados de Petróleo criou um comitê para padronizar a metodologia ASTM para São Paulo. Esse comitê formado por especialistas da CETESB, companhias de petróleo (BR, Ipiranga, Esso e Shell), empresas brasileiras de engenharia ambiental e consultores internacionais formalizaram em setembro de 2000 o procedimento: Ações Corretivas Baseadas em Risco Aplicadas a Áreas Contaminadas com Hidrocarbonetos Derivados de Petróleo e Outros Combustíveis Líquidos (ACBR $)^{6}$.

Esse procedimento foi desenvolvido visando contribuir no processo de gerenciamento da contaminação em postos de serviços, bases de distribuição de combustíveis, terminais ou em outras áreas onde sejam manipulados e/ou armazenados compostos derivados de petróleo cru.

Em todos esses modelos, nacionais e internacionais, utilizam-se as propriedades físicas e químicas do composto para determinar seu comportamento no meio ambiente. Sabe-se que essas propriedades influenciam diretamente a mobilidade e persistência do contaminante no meio ambiente, daí a grande importância da utilização de valores, o mais representativo possível, de dados importantes como o coeficiente de distribuição $(\mathrm{Kd})$ nos modelos pré-estabelecidos. É importante ressaltar que apesar desses parâmetros já possuírem padrões obtidos em testes de campo e laboratório (em sua grande maioria realizada no exterior), eles são muito sensíveis ao tipo e composição do combustível, ao meio (tipo de solo) onde ocorreu o vazamento e às condições ambientais (ASTM) ${ }^{7}$. Portanto, são necessários estudos mais detalhados do comportamento desses contaminantes em solo brasileiro, para melhor adequação dos modelos pré-existentes às condições climáticas e ambientais brasileiras.

\section{PARTE EXPERIMENTAL}

\section{Reagentes e soluções}

Todos os reagentes empregados no estudo eram de grau analítico e as soluções foram preparadas com água ultrapura do sistema Millipore Milli-Q. Os ensaios de isotermas foram realizados à temperatura de $25{ }^{\circ} \mathrm{C}$. As soluções estoques de benzo(a)pireno foram preparadas a partir da diluição de solução padrão $\left(500 \mathrm{mg} \mathrm{L}^{-1}\right)$, preparada a partir do sólido do composto. A quantificação do benzo(a)pireno foi realizada através de cromatografia a gás acoplada a espectrômetro de massas, utilizando-se como método referência o publicado pela Agência de Proteção Ambiental Americana (U.S.EPA 8270C) ${ }^{8}$.

\section{Preparação das isotermas de sorção}

Os ensaios de isotermas de sorção foram realizados em triplicata para as duas amostras de solo do horizonte A $(0-10 \mathrm{~cm})$ e horizonte B (1,0-1,1 m), conforme descrito a seguir: seis alíquotas de $1 \mathrm{~g}$ de cada solo estudado foram pesadas em balança analítica e transferidas para um tubo de centrífuga de $50 \mathrm{~mL}$. Tomou-se o cuidado de quartear a amostra antes da pesagem, garantindo assim sua representatividade; a cada uma das amostras (horizonte A e horizonte B) foram adicionadas alíquotas de seis diferentes concentrações de benzo(a)pireno, variando de 1,18 a 35,4 $\mathrm{mg} \mathrm{L}^{-1}$; aos tubos contendo solo e concentrações conhecidas de benzo(a)pireno adicionou-se solução 1:1 de água/ metanol completando um volume final de $20 \mathrm{~mL}$; cada tubo, contendo a amostra de solo e benzo(a)pireno em solução de metanol/água (1:1), foi mantido sob agitação a $200 \mathrm{rpm}$ durante $24 \mathrm{~h}$ à $25^{\circ} \mathrm{C}$; o tubo foi centrifugado a $3000 \mathrm{rpm}$ por $15 \mathrm{~min}$; uma alíquota de aproximadamente $10 \mathrm{~mL}$ do sobrenadante foi retirada, filtrada através de um sistema de filtração Millipore com membrana filtrante de $0,45 \mu \mathrm{m}$; na alíquota filtrada determinou-se a concentração do benzo(a)pireno da solução após o período de $24 \mathrm{~h}$.

As concentrações de benzo(a)pireno adicionadas (Co) ao ensaio de sorção e a média da concentração de benzo(a)pireno (Ce) após 24 h de agitação foram utilizadas para cálculo da quantidade de BAP sorvida (qe) ao solo e para construção das isotermas de sorção apresentadas na Figura 1.

\section{Amostras de solo}

As amostras de solo do horizonte A (profundidade de 0 a $10 \mathrm{~cm}$ ) e horizonte B (profundidade de 1,0 a 1,10 m) foram coletadas na região de Sorocaba, estado de São Paulo, em uma área pouco impactada por contaminações ambientais. Suas características são apresentadas na Tabela 1 .

\section{RESULTADOS E DISCUSSÃO}

\section{Avaliação das isotermas de sorção}

Uma avaliação das curvas de sorção (Figura 1) mostra que as curvas obtidas pelos ensaios de sorção não seguiram a tendência da maioria das curvas apresentadas na literatura. A maioria dos estudos de sorção para compostos não polares tem observado isotermas do tipo L (Langmuir) ou C (partição constante) ${ }^{9-13}$. As isotermas obtidas neste estudo para BAP indicam uma tendência à isoterma de sorção do tipo $\mathrm{S}$ (sigmoidal). A definição das isotermas de sorção $\mathrm{L}, \mathrm{C}, \mathrm{H}$ e $\mathrm{S}$ é discutida por Giles ${ }^{14}$. 

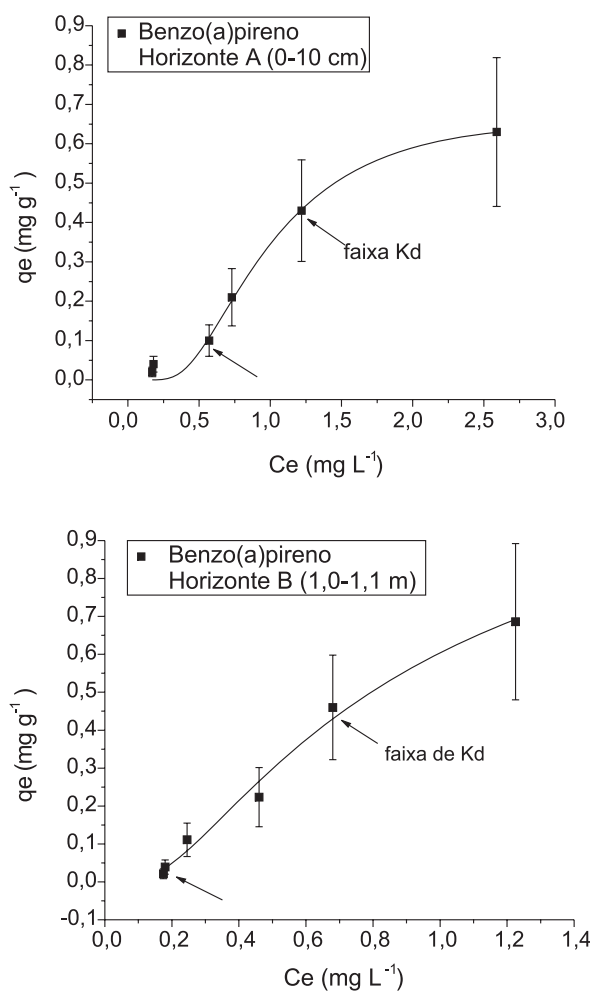

Figura 1. Curva de sorção do Benzo(a)pireno-Amostras de solo do horizonte A $(0-10 \mathrm{~cm})$ e horizonte $B(1,0-1,1 \mathrm{~m})$

Tabela 1. Resultados de caracterização físico-química das amostras de solo

\begin{tabular}{lcc}
\hline Parâmetro & $\begin{array}{c}\text { Horizonte A } \\
(0-10 \mathrm{~cm})\end{array}$ & $\begin{array}{c}\text { Horizonte B } \\
(1,0-1,1 \mathrm{~m})\end{array}$ \\
\hline Granulometria & & \\
Areia & $60 \%$ & $48 \%$ \\
Silte & $20 \%$ & $22 \%$ \\
Argila & $20 \%$ & $30 \%$ \\
pH & 4.2 & 4.0 \\
CTC & $6,4 \mathrm{cmol} \mathrm{kg}-1$ & $6,4 \mathrm{cmol} \mathrm{kg}^{-1}$ \\
Matéria orgânica & $1,72 \%$ & $1,80 \%$ \\
\% Carbono orgânico & $0,997 \%$ & $1,043 \%$ \\
& (foc: 0,00997$)$ & (foc: 0,01043$)$ \\
\hline
\end{tabular}

CTC: Capacidade de Troca Catiônica

Um levantamento mais detalhado da literatura mostrou que vários pesquisadores também observaram isotermas de sorção do tipo-S para compostos orgânicos não polares ${ }^{15-17}$. Mas até hoje, ainda não foi estabelecido um conceito claro para explicar o comportamento de interação de compostos orgânicos não polares com o solo. Vários autores sugerem alguns mecanismos de interação e comportamentos das curvas de sorção para compostos orgânicos não polares. Porém, a maioria dos estudos realiza os experimentos em meio aquoso e nem sempre utilizaram como adsorvedor um solo natural, mas sim argilominerais específicos ou ácido húmico puro, que, na realidade não representam fielmente o solo natural. É, portanto, muito difícil comparar os resultados deste trabalho, onde se estudou um solo natural e experimentos de sorção realizados em meio água/metanol (1:1), com trabalhos da literatura.

A maioria dos pesquisadores concorda que o comportamento de retenção de compostos orgânicos no solo é fortemente depen- dente da matéria orgânica do solo. Bobe et al. ${ }^{18}$ observaram que sistemas de sorção de solos com baixos teores de matéria orgânica tendem a gerar isotermas tipo-S. De acordo com Stevenson ${ }^{19}$ o ácido húmico é o constituinte da matéria orgânica que é mais insolúvel e tende a se fixar à superfície das partículas inorgânicas do solo (principalmente argilominerais, devido a sua área específica), modificando os pontos de sorção para os poluentes orgânicos. Ele também afirma que isotermas tipo-S ocorrem normalmente quando o sistema adsorvedor (solo) tem elevada afinidade com o solvente. De acordo com Young e Weber ${ }^{20}$, a matéria orgânica do solo é considerada heterogênea e consiste de componentes "hard" e "soft" (Figura 2), que exibem comportamentos de sorção diferentes para compostos orgânicos. Os componentes mais acessíveis são denominados matéria orgânica tipo borracha ("soft" ou "rubbery") e os componentes menos acessíveis são denominados rígidos ou vítreos ("hard" ou "glassy") que exibem comportamentos de sorção diferentes. Segundo $\mathrm{Chiou}^{21}$, o poluente orgânico pode ser sorvido superficialmente pela matéria orgânica aderida à fração mineral ou à fração orgânica solúvel. Em uma segunda etapa, o poluente orgânico pode difundir para dentro dos mesoporos e microporos e interagir com a matéria orgânica "rubbery" ou "soft" (microporos mais acessíveis) ou "glassy" e "hard" (microporos menos acessíveis). As maiorias dos adsorvedores são corpos altamente porosos, com área superficial interna muito grande. A superfície externa constitui somente uma pequena fração da superfície total do adsorvedor. Em geral, uma sorção não linear é resultado do processo de sorção na fração "hard" da matéria orgânica do solo.

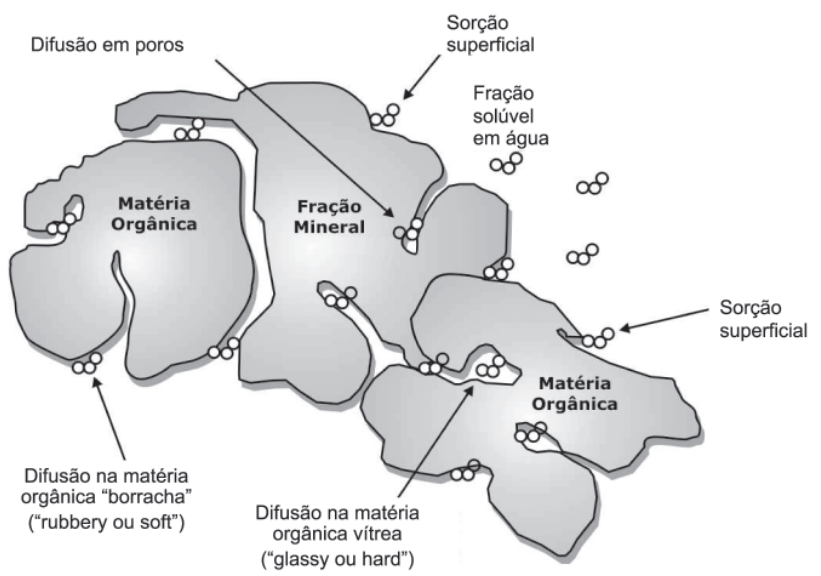

Figura 2. Comportamento do contaminante orgânico no solo. Adaptado da ref. 22

Por outro lado, como os ensaios de sorção foram conduzidos em meio água/metanol (1:1), provavelmente um efeito de cosolvência foi favorecido. Denomina-se efeito de cosolvência o efeito causado por um determinado solvente orgânico sobre a solubilidade e sorção de compostos orgânicos hidrofóbicos. Rao et al. ${ }^{23}$ afirmaram que com o aumento do volume de solvente orgânico utilizado em uma mistura de solvente binária, há um aumento da solubilidade dos contaminantes orgânicos hidrofóbicos e diminui a sorção desses compostos ao solo. Além disso, cosolventes também podem alterar a natureza hidrofóbica da fração orgânica do solo, aumentando o teor de carbono orgânico dissolvido na fase solução, alterando desta forma todo o comportamento de contaminantes hidrofóbicos no solo.

HPAs são compostos hidrofóbicos de baixa solubilidade em água e, portanto, tendem a se ligar à matéria orgânica do solo, limitando sua disponibilidade. A presença de metanol (efeito de cosolvência) irá aumentar a solubilidade dos HPAs e, portanto, sua 
Tabela 2. Comparação dos valores do coeficiente de distribuição (Kd) de benzo(a)pireno calculados com base nos dados de Koc do EPA ${ }^{3}$ e do relatório holandês ${ }^{4}$ com os dados obtidos experimentalmente das amostras de solo do horizonte A $(0-10 \mathrm{~cm})$ e horizonte B (1,0-1,1m)

\begin{tabular}{|c|c|c|c|c|c|c|c|c|}
\hline Benzo(a)pireno & $\begin{array}{c}\text { Koc } \\
\text { Min.-máx } \\
\left(x 10^{3} \mathrm{~L} \mathrm{~kg}^{-1}\right)\end{array}$ & $\begin{array}{l}\text { Número de } \\
\text { dados } \\
\text { publicados }\end{array}$ & $\begin{array}{c}\text { Kd calculado } \\
* * * * \\
\text { Min-max } \\
\text { Horizonte A } \\
\left(\mathrm{x} 10^{3} \mathrm{~L} \mathrm{~kg}^{-1}\right)\end{array}$ & $\begin{array}{c}\text { Kd calculado } \\
* * * * \\
\text { Min-max } \\
\text { Horizonte B } \\
\left(\mathrm{x} 10^{3} \mathrm{~L} \mathrm{~kg}^{-1}\right)\end{array}$ & $\begin{array}{c}\text { Kd experimental } \\
\text { Horizonte A } \\
\left(\mathrm{x} 10^{3} \mathrm{~L} \mathrm{~kg}^{-1}\right)\end{array}$ & $\begin{array}{c}\text { Fator } \\
\text { Calc/exp. } \\
\text { Horizonte A }\end{array}$ & $\begin{array}{c}\text { Kd experimental } \\
\text { Horizonte B } \\
\left(\mathrm{x} 10^{3} \mathrm{~L} \mathrm{~kg}^{-1}\right)\end{array}$ & $\begin{array}{c}\text { Fator } \\
\text { Calc/exp. } \\
\text { Horizonte B }\end{array}$ \\
\hline $\mathrm{EPA}^{3}$ & $479-2130$ & 3 & $4,8-21,6$ & $4,9-22$ & $0,52 \pm 0,18$ & $9-42$ & $0,83 \pm 0,32$ & $6-27$ \\
\hline Bockting et al. ${ }^{4}$ & $77-478$ & 2 & $0,774-4,8$ & $0,810-4,9$ & $0,52 \pm 0,18$ & $2-9$ & $0,83 \pm 0,32$ & $1-6$ \\
\hline
\end{tabular}

$* * * * \mathrm{Kd}=$ Koc. foc. Kd calculado a partir do Koc da ref. 3 e ref. 4. foc experimental horizonte A = 0,00997 e foc experimental horizonte $\mathrm{B}$ $=0,01043$ (Tabela 1$)$.

disponibilidade no solo. Estudos do comportamento de compostos orgânicos hidrofóbicos realizados em soluções contendo solvente orgânico representam melhor a realidade, já que estes composto (HPAs) são comumente encontrados em contaminações industriais (ex.: postos de combustíveis) na presença de solventes orgânicos .

\section{DETERMINAÇÃO DE COEFICIENTE DE DISTRIBUIÇÃO (KD) PARA O BENZO(A)PIRENO}

O coeficiente de distribuição Kd foi obtido através da porção linear da isoterma de sorção do BAP, denominada faixa de $\mathrm{Kd}$ apontadas na Figura 1. O Kd foi determinado pela relação $\Delta \mathrm{qe}\left(\mathrm{mg} \mathrm{kg}^{-1}\right) / \Delta \mathrm{Ce}$ $\left(\mathrm{mg} \mathrm{L}^{-1}\right)$ e o valor obtido para o Horizonte A foi de $0,52 \times 10^{3}$ e para o Horizonte B de $0,83 \times 10^{3}\left(\mathrm{~L} \mathrm{~kg}^{-1}\right)$ (Tabela 2). Para efeito de avaliação dos dados obtidos, os valores de $\mathrm{Kd}$ experimentais foram comparados com os valores de $\mathrm{Kd}$ calculados a partir de valores de Koc da literatura $\mathrm{EPA}^{3}$ e dados do relatório holandês ${ }^{4}$.

Avaliando-se a Tabela 2 observa-se que o valor calculado de Kd é maior que o valor de Kd obtido experimentalmente. Relacionando-se os valores de $\mathrm{Kd}$ calculados com os valores obtidos experimentalmente neste trabalho para o BAP, obtém-se um fator que apresenta grande variação quando comparado com os valores referenciados pela Agência de Proteção Ambiental Americana, chegando a 42 quando comparado com o valor máximo.

Observa-se também que os valores calculados de Kd com base na literatura holandesa também são maiores que os valores obtidos experimentalmente. Na razão de Kd calculado versus experimental, observa-se que o fator é inferior a 6 para as duas amostras estudadas.

A seguir serão discutidas as possíveis razões que afetaram o valor de Kd obtido experimentalmente a ser inferior ao valor encontrado na literatura.

\section{Variação dos valores máximos e mínimos do Koc}

Os valores de Koc publicados pela EPA e apresentados na Tabela 2 foram obtidos com base em revisão da literatura e mostram que estes dados apresentam grande variação entre si, muitas vezes em várias ordens de grandeza. Essa variação pode ser atribuída a vários fatores, que segundo a EPA podem estar relacionados à: diferenças nas propriedades das amostras de solo; diferenças nos ensaios e método analítico utilizado, e erros experimentais ou de medidas. $\mathrm{O}$ mesmo ocorre com os valores do relatório holandês de Koc, onde também são poucos os dados de Koc usados para o cálculo do valor médio publicado.

\section{Escassez de literatura sobre HPAs em solo}

Verificou-se também que na literatura poucos foram os estu- dos experimentais realizados para obtenção de Koc em compostos orgânicos, objeto desse trabalho (benzo(a)pireno). É surpreendente que os valores de Koc para compostos orgânicos não ionizáveis, grupo onde se inclui o benzo(a)pireno, sejam muito pequenos, principalmente para este composto cujo grau de toxicidade e fator carcinogênico é muito elevado. Essa escassez de dados experimentais de Kd ou Koc mostra a dificuldade desse trabalho e a complexidade da pesquisa desses coeficientes.

\section{Escassez de estudos de HPAs em solo natural}

Grande parte dos dados apresentados, tanto pelo EPA ${ }^{3}$ quanto por Bockting et $a l .{ }^{4}$ foram obtidos por experimentos realizados em amostras de ácidos húmicos puros, sedimentos e argilominerais específicos ao invés de um solo natural. Portanto, estudar e determinar estes coeficientes (Kd e Koc) em amostras de solo natural, que apresentam uma combinação de sítios sorvedores (como argilominerais e ácidos húmicos) é muito mais complexo, e daí a provável razão do alto grau de incerteza obtido na comparação dos dados de Kd deste trabalho com a literatura. Além disso, existem poucas referências na literatura sobre experimento de Koc realizados em amostras de solo natural e praticamente não existem referências de experimentos em amostras de solo tropical.

\section{Características hidrofóbicas}

Outra razão para a provável incerteza nos valores experimentais de Kd do benzo(a)pireno em relação aos valores da literatura provem da sua alta hidrofobicidade. A propriedade hidrofóbica favorece a sorção dos compostos orgânicos à matéria orgânica do solo. As características húmicas do solo apresentam complexidade estrutural e composição química pouco definida e, portanto, as interações entre o benzo(a)pireno e a matéria húmica do solo podem ocorrer por diferentes processos, ainda não completamente conhecidos.

\section{Efeito de cosolvência}

Solventes orgânicos agem com cosolventes de contaminantes orgânicos no meio ambiente. Denomina-se efeito cosolvência o efeito de um determinado solvente orgânico, completamente miscível em água, agir na solubilidade e sorção de compostos orgânicos hidrofóbicos.

A justificativa mais provável e relevante para a obtenção de $\mathrm{Kd}$ experimentais muito menores que os calculados pela literatura é devido à utilização do cosolvente metanol nos ensaios de isotermas de sorção do presente trabalho. Uma análise dos dados de Koc da literatura ( $\mathrm{EPA}^{3}$ e Relatório holandês $\left.{ }^{4}\right)$ mostra que somente um experimento foi realizado neste meio. $\mathrm{O}$ metanol atua como cosolvente 
e pode alterar a solubilidade dos compostos orgânicos e a natureza hidrofóbica da fração orgânica do solo, aumentando o teor de carbono orgânico dissolvido na fase solução alterando, desta forma, todo o comportamento dos HPAs no solo ${ }^{21}$.

Da discussão acima concluiu-se que apesar dos poucos dados disponíveis na literatura, e da quase inexistência de ensaios para solo natural, o fator que mais contribuiu para a obtenção de $\mathrm{Kd}$ experimentais inferiores aos da literatura foi o efeito da cosolvência.

\section{PORCENTAGEM DE SORÇÃO DO BENZO(A)PIRENO NO SOLO}

Como parte do trabalho de determinação do Kd do benzo(a)pireno no solo estudado, foram calculadas as porcentagens de sorção (\% sorção $=\mathrm{Cad} * 100 / \mathrm{Co})$ da amostra horizonte A e comparadas com os resultados do horizonte $\mathrm{B}$.

A Figura 3 apresenta os resultados deste estudo.

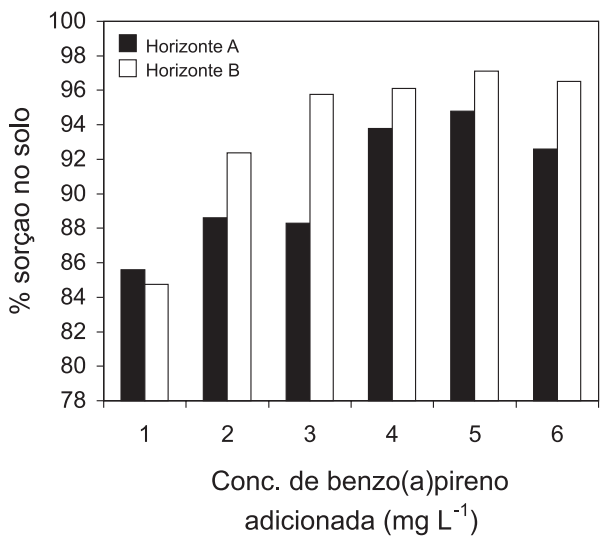

Figura 3. Porcentagens de sorção benzo(a)pireno nas amostras de solo do horizonte $A(0-10 \mathrm{~cm})$ e horizonte $B(1,0-1,1 \mathrm{~m})$, variando a concentração adicionada de BAP de $1,18 \mathrm{mg} \mathrm{L}^{-1}(1)$ a $35,4 \mathrm{mg} \mathrm{L}^{-1}$

Avaliando a Figura 3, observou-se que o benzo(a)pireno apresentou uma alta porcentagem de sorção na amostra de solo em estudo, com valores variando de 84,7 a $97,1 \%$ de sorção.

A Figura 3 mostra que há um acréscimo na porcentagem de sorção do benzo(a)pireno da amostra do horizonte A $(0-10 \mathrm{~cm})$ em relação à amostra horizonte $\mathrm{B}(1,0-1,1 \mathrm{~m})$, provavelmente devido ao aumento da porcentagem de argila da amostra do horizonte A para B (Tabela 1). A interação dos HPAs com o solo ocorre através da matéria orgânica aderida às partículas do solo e essa interação será maior quanto maior for a superfície de contato. Esses dados são consistentes com pesquisas realizadas por $\operatorname{Pichler}^{24}$, que encontrou grandes variações na concentração de HPAs em diferentes frações granulométricas do solo. A concentração do BAP aumentou com o decréscimo do tamanho das partículas.

\section{CONCLUSÕES}

O estudo de determinação do coeficiente de distribuição (Kd) de benzo(a)pireno em solo mostrou que a curva de sorção não é linear e apresenta forma tipo-S, provavelmente por causa das condições experimentais do sistema solo-água-metanol; o cálculo do $\mathrm{Kd}$ foi possível pela curva de sorção. O valor do Kd experimental apre- sentou valores menores que o valor do Kd calculado a partir de Koc da literatura. O fator que mais contribuiu para a obtenção de Kd experimental inferior ao da literatura foi o efeito cosolvência; o cálculo da porcentagem de sorção mostrou que a argila pode contribuir para um aumento da sorção de benzo(a)pireno ao solo, provavelmente por causa do aumento da área superficial, que favoreceu a adsorção da matéria orgânica ao argilomineral e a sorção do benzo(a)pireno à matéria orgânica.

O cálculo do valor de Kd é um parâmetro muito importante para os modelos de avaliação de risco. Portanto, quando se inicia uma análise de risco e se define o Kd a ser aplicado ao modelo, deve-se primeiramente avaliar o composto orgânico a ser utilizado. Em uma segunda etapa deve-se avaliar a porcentagem de matéria orgânica presente no solo em estudo e também a granulometria, principalmente a porcentagem de argila pode contribuir para o aumento de área específica da matéria orgânica aderida ao argilomineral e possibilitar maior interação do benzo(a)pireno ao solo. Um outro fator que deve ser cuidadosamente examinado é a presença de cosolvente no caso de estudo de contaminações por poluentes orgânicos em solos. O estudo de interação de BAP em sistema água-metanolsolo mostrou que pode ocorrer uma redução do valor de Kd neste meio, isto é, a presença de um cosolvente aumenta a disponibilidade dos contaminantes orgânicos no solo e, portanto, favorece a contaminação do aquífero.

\section{REFERÊNCIAS}

1. Blümer, M.; Science 1961, 134, 474.

2. CETESB, Companhia de Tecnologia de Saneamento Ambiental; Relatório de estabelecimento de Valores Orientadores para Solos e Águas Subterrâneas no Estado de São Paulo. Relatório Técnico, 2001.

3. http://www.epa.gov/oerrpage/superfund/resources/soil/toc.htm, acessada em Janeiro 2004.

4. Bockting, G. J. M.; van de Plassche, E. F.; Struijs, J.; Canton, J. H.; Report no.: 679101013 National Institute of Public Health and Environmental Protection Bilthoven, The Netherlands, 1993.

5. Kehew, A. E.; Applied Chemical Hydrogeology, Prentice-Hall, Inc: Upper Saddle River, New Jersey, 2001.

6. ACBR - Câmara Ambiental do Comércio de Derivados de Petróleo; Versão Preliminar, setembro/2000.

7. ASTM-PS-104: Standard Provisional Guide for Risk-Based Corrective Action, 1998.

8. http://www.epa.gov/epaoswer/hazwaste/test/pdfs/8270c.pdf, acessada em Julho 2004.

9. Karickhoff, S. W.; Brown, D. S.; Scott,T. A.; Water Res. 1979, 13, 241.

10. Means, J. C.; Wood, S. G.; Jhassett, J. J.; Banwast, W. L.; Environ. Sci. Technol. 1980, 14, 1524.

11. McCarthy, J. F.; Jimenez, B. D.; Binding and Dissociation 1985, 19, 1072.

12. Magee, B. R.; Leonard W. L.; Lemley, A. T.; Environ. Sci. Technol. 1991, $25,323$.

13. Rav-Acha, C. H.; Rebhun, M.; Water Res. 1992, 26, 1645.

14. Giles, C. H.; Smith, D; Huitson, A.; J. Colloid Interface Sci. 1974, 47, 755.

15. Keith, L. H.; Telliard, W. A.; Environ. Sci. Technol. 1979, 13, 416.

16. Gunasekara, A. S.; Xing, B.; J. Environ. Qual. 2003, 32, 240.

17. Mao, J. D.; Hundal, S.; Thompson, M. L.; Schmidt-Rohr, K.; Environ. Sci. Technol. 2002, 36, 929.

18. Bobe, A.; Coste, C. M.; Cooper, J. F.; J. Agric.Food Chem. 1997, 45, 4861.

19. Stevenson, F. J.; Humus Chemistry. Genesis, composition reactions, $2^{\text {nd }}$ ed., John Wiley \& Sons: New York. 1994.

20. Young, T. M.; Weber, W. J.; Environ. Sci. Technol. 1995, 29, 92.

21. Choiu, C.T.; Partition and adsorption of organic contaminants in environmental systems, $1^{\text {st }}$ ed., Wiley: New Jersey, 2002.

22. Semple, K. T.; Morriss, W.;Paton, G. I.; European Journal of Soil Science 2003, 54, 809.

23. Rao, P. S. C.; Lee, S. L.; Pinal, R.; Environ. Sci. Technol. 1990, 24, 647.

24. Pichler, M.; Bayreuther Bodenkunde Bericht 1995, 46, 108 